\title{
EL DERECHO A LA SALUD SEXUAL Y REPRODUCTIVA EN LA PANDEMIA DEL COVID-19 Y LA VIOLENCIA ESTRUCTURAL DE GÉNERO
}

\author{
THE RIGHT TO SEXUAL AND REPRODUCTIVE HEALTH IN THE COVID-19 PANDEMIC AND \\ STRUCTURAL GENDER-BASED VIOLENCE
}

\author{
Lupe Vargas-Zafra (iD) 1,a
}

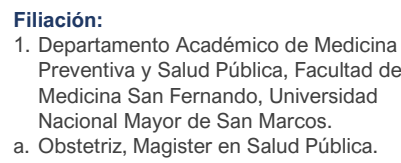

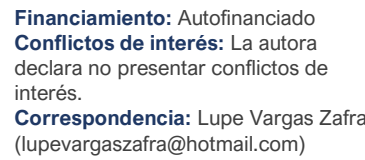

Financiamiento: Autofinanciado Conflictos de interés: La autora declara no presentar conflictos de interés.

Correspondencia: Lupe Vargas Zafra (lupevargaszafra@hotmail.com)

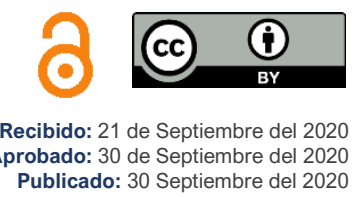

Los Objetivos de Desarrollo Sostenible 3. "Salud y Bienestar" y 5. "Lograr la igualdad de género y empoderar a todas las mujeres y las niñas", reconocen que la buena salud es esencial para el desarrollo sostenible. Para lograrlo establecen como objetivo poner fin a todas las formas de discriminación contra las mujeres y niñas, considerándolo como un derecho humano básico y crucial. El objetivo 5 plantea como una de las metas: lograr que las mujeres de 15 a 49 años tomen sus propias decisiones informadas sobre relaciones sexuales, el uso de anticonceptivos y atención de la salud reproductiva. En el Perú, para lograrlo, se genera la Ley $N^{\circ} 28983$, Ley de igualdad de oportunidades entre hombres y mujeres (1); esta Ley establece la adopción de políticas, planes y programas para su implementación. Es así como la Política General de Gobierno al año 2021 (2), incluye un lineamiento prioritario orientado a promover la igualdad y no discriminación entre hombres y mujeres, así como garantizar la protección de la niñez, la adolescencia y las mujeres frente a todo tipo de violencia. En este contexto, se aprueba la Política Nacional de Igualdad de Género, para abordar las causas y los efectos de la discriminación estructural contra las mujeres, encomendándole su implementación al Ministerio de la Mujer y Poblaciones Vulnerables (3).

La discriminación estructural, es el conjunto de prácticas reproducidas por patrones socioculturales instalados en las personas, las instituciones y la sociedad en general. Tiene como uno de los efectos a la vulneración de los derechos a la salud sexual y salud reproductiva, que se expresa en prácticas excluyentes y violentas, quebrantando así el derecho humano a una vida libre de violencia. El Índice de Desigualdad de Género (IDG) mide las brechas de género en el Perú, aborda la problemática de género en el aspecto de la salud sexual y reproductiva como una variable importante del desarrollo humano, junto a otros aspectos como el empoderamiento y la participación en la fuerza laboral. (INEI, 2017b, 17-18). En el aspecto de la salud sexual y reproductiva se consideran a los siguientes indicadores para evaluar su estado: tasa de mortalidad materna, el acceso a los métodos modernos de planificación familiar y el embarazo en adolescentes.

La situación social y económica causada por la pandemia del COVID-19 en el Perú ha generado incremento de la desigualdad y desventaja de las mujeres para asumir sus derechos sexuales y reproductivos, situación que se evidencian con el incremento con la violencia física y psicológica, el embarazo en adolescentes y la muerte materna (4). Las adolescentes están en situación de vulnerabilidad ante escenarios de violencia física, sexual y psicológica; un porcentaje considerable de ellas quedan embarazadas limitando la oportunidad de seguir desarrollándose en la vida, sobre todo si residen en zonas de pobreza y excusión. Las brechas de género entre hombres y mujeres son evidentes con mayor frecuencia, en las regiones que estructuralmente son más pobres, donde los servicios de salud pública no llegan de manera oportuna, adecuada y diferenciada. Ante esta situación es 
momento de dejar el discurso teórico y poner en práctica los servicios públicos, sobre todo la atención de la salud a las mujeres, para revertir los siguientes indicadores, de manera inmediata.

En el presente año, hasta la semana epidemiológica 37, se han reportado 292 muertes maternas, 61 más que el año 2019. Los departamentos que tienen un incremento notorio de muertes maternas son Huancavelica (400\%), Arequipa (200\%), Cusco (167\%) y Tacna (100\%). En las regiones de Tumbes y Pasco, el año 2019 no se registró ninguna muerte materna, sin embargo, en el presente año se ha reportado 5 muertes en Tumbes y 2 muertes Pasco. Si se compara la muerte materna por grupo atareo, el incremento se produce en las mujeres que tienen más de 35 años, pasando de $26.2 \%$ en el año 2019 a $30.5 \%$ hasta la semana 37 del presente año. Según el reporte epidemiológico, también se observa un incremento de las muertes maternas indirectas de $33.1 \%$ en el año 2019 a $43.2 \%$ en lo que va del año 2020 (5).

Respecto al acceso al uso de métodos anticonceptivos para la planificación familiar, según reporte de la encuesta ENDES 2019, solo el $55.6 \%$ de las mujeres usan métodos modernos, del total de usuarias que usan algún método anticonceptivo. La desigualdad se hace más notoria en las residentes de la zona rural donde se limitan a usar métodos anticonceptivos tradicionales, mientras que las mujeres que viven en la zona urbana tienen mayor oportunidad de uso de los métodos modernos.

El embarazo en adolescentes sigue siendo un serio problema de salud pública, marcado por los determinantes estructurales de la salud, que afectan su desarrollo, más aún si viven en condiciones de pobreza. Según el Sistema de Registro del Certificado de Nacidos vivos del Ministerio de Salud, hasta setiembre del presente año, se registra que el $10 \%$ de nacimientos se produjeron en madres adolescentes en el Perú. Las regiones con mayor índice de pobreza son las que tienen mayor porcentaje de embarazo en adolescentes: Loreto con el $20 \%$; Ucayali con el $18 \%$; Huancavelica, con 18\%; San Martín, con el 16\%; y Cajamarca, con el $14 \%$ (6)

Por otro lado, la ENDES 2019 (7), al realizar la encuesta a mujeres de 15 a 49 años, para recoger información sobre la violencia familiar hacia las mujeres en el País, reportó que el $57,7 \%$ de mujeres fueron víctimas de violencia ejercida alguna vez por el esposo o compañero; situación que se manifiesta más en las familias redientes en la zona urbana $(58,3 \%)$ que en la zona rural $(55,3 \%)$.

La discriminación estructural contra las mujeres en el Perú es un problema público que requiere la adopción de medidas adecuadas y oportunas de diversos sectores que trabajen los determinantes estructurales. Desde el Sector Salud de deben establecer protocolos de acceso a los servicios de salud sexual y reproductiva considerando los lineamientos emitidos en las políticas que se enfocan a disminuir las brechas de género, se garantizará, de esta manera uno de los derechos fundamentales de las mujeres y sus familias que es el acceso a los servicios de salud, a la atención y recuperación en salud, el acceso a la información, protección y el consentimiento informado. Es urgente revertir la opinión que expresan las mujeres al ser encuestadas, donde el $90.6 \%$ manifiestan que tienen problemas de acceso a servicios de salud por los siguientes motivos: "que no había medicamentos" ( $80,7 \%)$, que "no había algún personal de salud" (76,9\%), y por problemas económicos para el tratamiento $(50,3 \%)(7)$.

La implementación del enfoque de género en las políticas públicas como una herramienta para trabajar las oportunidades de igualdad de derechos, roles y tareas que realizan los hombres y las mujeres en una sociedad, es crucial para el desarrollo del país, sin embargo, por sí solas no son suficientes para cambiar las prácticas institucionales, para este tema, de los servicios de salud a favor de las mujeres (8). Para lograr el acceso a los servicios de salud, debe asegurarse que el discursos no solo termine en la teoría al mencionar el reforzamiento del primer nivel de atención, sino establecer una respuesta rápida a la enfermedad causada por el COVID-19, incluidos los servicios de salud sexual y reproductiva, con particular atención a las mujeres embarazadas, los y las adolescentes y poblaciones vulnerables; intervenciones que también deben ser realizadas con criterio de interseccionalidad hacia las personas que pertenecen a grupos indígenas y aquellas en situación de pobreza. 


\section{REFERENCIAS}

1. Diario El Peruano. Ley $\mathrm{N}^{\circ}$ 28983: Ley de igualdad de oportunidades entre hombres y mujeres. Lima, Perú. 2007. [Link]

2. Diario El Peruano. Decreto Supremo $\mathrm{N}^{\circ}$ 056-2018-PCM, que aprueba la Política General de Gobierno al 2021. Lima, Perú. 2018. [Link]
3. Diario El Peruano. Política Nacional de Igualdad de Género, aprobada mediante Decreto Supremo $\mathrm{N}^{\circ} 008$ 2019-MIMP. Lima, Perú. 2019. [Link]

4. Resúmenes Técnicos del UNFPA. Los adolescentes y los jóvenes y la enfermedad del Coronavirus (COVID19). 2020. [Link]

5. Ministerio de Salud. Sala situacional para el análisis de la situación de salud. Lima, Perú. 2020. [Link]
6. Ministerio de Salud. Sistema del Registro del Certificado de Nacido Vivo, REUNIS Lima, Perú. 2020. [Link]

7. Instituto Nacional de Estadística e Informática. Encuesta Nacional de Hogares. Lima, Perú. 2019. [Link]

8. Resúmenes Técnicos del UNFPA. La Salud y los Derechos Sexuales y Reproductivos, la Salud Materna y Neonatal y la COVID-19. 2020. [Link] 